\title{
Innovative Design on Digital Jacquard with 2:1 Weft-Backed Structure
}

\author{
$\mathrm{Xi}$ PENG and Jiu ZHOU ${ }^{1}$ \\ Silk and Fashion Culture Research Center of Zhejiang Province, Zhejiang Sci-Tech \\ University
}

\begin{abstract}
In order to inherit and innovate traditional weft-backed structure jacquard fabric, a design principle and method of digital jacquard with 2:1 weftbacked structure was proposed. Based on the in-depth analysis of the characteristics of the traditional 2:1 weft-backed structure, four digital structural models (A1, A2, B1 and B2) with shaded weave were built by using digital jacquard technology. It lays a theoretical foundation for the creation of four kinds of digital jacquard fabrics with colour shading effects. In addition, the combined application of model A1 and model B1 was taken as an example to illustrate the design method. The resulting fabric had a delicate and natural colour shading effect. And its main and minor motifs were clearly distinguished. Meanwhile, the colour expression was increased while the yarn utilisation and production efficiency were improved. The principle and method proposed in this paper can create a wide variety of digital jacquard fabrics with colour shading effects, which provides ideas for the structural innovation and variety design of traditional jacquard fabrics.
\end{abstract}

Keywords. Digital jacquard, structural model, weft-backed structure, colour shading effect, weave database

\section{Introduction}

Jacquard fabric with weft-backed structure is a kind of traditional fabric woven by at least 1 set of warps and 2 sets of wefts, and the weft arrangement normally is $1: 1,2: 1$, $3: 1$, etc. The jacquard fabric with $2: 1$ weft-backed structure is a weft-backed structure fabric and its weft arrangement is $2: 1$. Due to the restrictions of traditional single plane design model, there are only a maximum of 18 colours on the traditional jacquard fabric with 2:1 weft-backed structure [1-2]. With the rapid development of digital jacquard technology, new opportunities arose to create digital jacquard fabric with 2:1 weft-backed structure. At present, digital research mainly focuses on the fabrics with 1:1 weft-backed structure [3-4], but has not yet been carried out on the fabrics with 2:1 weft-backed structure. The innovation of the weave structure is the key to creating digital jacquard fabric with 2:1 weft-backed structure. In order to solve these problems, this paper analysed the characteristics of the traditional 2:1 weft-backed structure, and created four digital structural models (A1, A2, B1 and B2) which combined two traditional structural models (A and $\mathrm{B}$ ) with a shaded weave by digital jacquard

1 Jiu ZHOU, Silk and Fashion Culture Research Center of Zhejiang Province, Zhejiang Sci-Tech University, No. 928, No. 2 Street, Xiasha High Education Park, Hangzhou, Zhejiang Province, China; Email: zhoujiu34@126.com. 
technology. In addition, the combined application of model A1 and B1 was taken as an example to illustrate the design method. It provides reference for the digital innovation of traditional jacquard fabrics.

\section{Digital design principle for jacquard fabric with $2: 1$ weft-backed structure}

The key to creating digital jacquard fabrics with colour shading effects is the combination of the $2: 1$ weft-backed structure with a shaded weave by digital jacquard technology.

\subsection{Design principle for traditional structural models}

2:1 weft-backed structure is formed by 1 set of warps and 2 sets of wefts (Wefts A and B). Wefts $A$ and $B$ are arranged in a 2:1 ratio. According to the covering principle of weft-backed structure [5], 2:1 weft-backed structure is composed of face weave and backing weave. As face weft covers backing weft, only the colour information of the face weft is shown on the face of the fabric. Both Wefts A and B can be used as face weft to show colours on the face, so 2 colours can be shown in the 2:1 weft-backed structure. The structure models and design principle are shown in Figure 1 and Figure 2 respectively. In model A, weave A is face weave and weave B is backing weave. As Wefts B is fully covered by Wefts A, only the colour of Wefts A is shown on the face of the fabric (Figure 2 (a)). To achieve the above covering effect, in the fully-backed structure A, the upper and lower rows (the position of Wefts A) corresponding to the weft points designed for any row of Wefts B must be weft points (Figure 2 (a)). In model B, weave B is face weave and weave A is backing weave. As Wefts A is fully covered by Wefts B, the colour of Wefts B is shown on the face (Figure 2 (b)). In the fully-backed structure B, the upper or lower row (the position of Wefts B) corresponding to the weft points designed for any row of Wefts A must be weft points (Figure 2 (b)).

\begin{tabular}{|l|l|l|}
\hline Structure model & Model A & Model B \\
\hline Face side & & \\
\hline Reversed side & & \\
Notes: & & \\
Weft A; & & \\
Weft B
\end{tabular}

Figure 1. Traditional structural models of 2:1 weft-backed structure.

\subsection{Design principle for digital structure models}

To create digital jacquard fabrics with colour shading effects, we should achieve not only a fabric covering effect, but also a colour shading effect. Shaded weave is a series of weaves which can achieve a color shading effect [6]. By adding the colour shading effect to face weave A, face weave A and backing weave B, face weave B, face weave $\mathrm{B}$ and backing weave $\mathrm{A}$ respectively in the traditional structural models, four digital structure models A1, A2, B1 and B2 can be obtained to achieve four kinds of colour shading effects on the fabric respectively. The design process of four digital structural models is shown in Figure 3. 


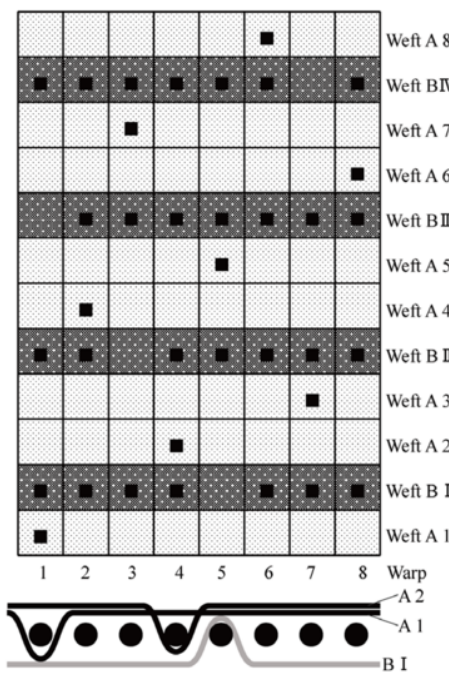

(a) Full-backed structure $\mathrm{A}$ Notes: $\square$ Warp/Weft A; 2 Warp/Weft B:

Warp; - Weft A; - Weft B

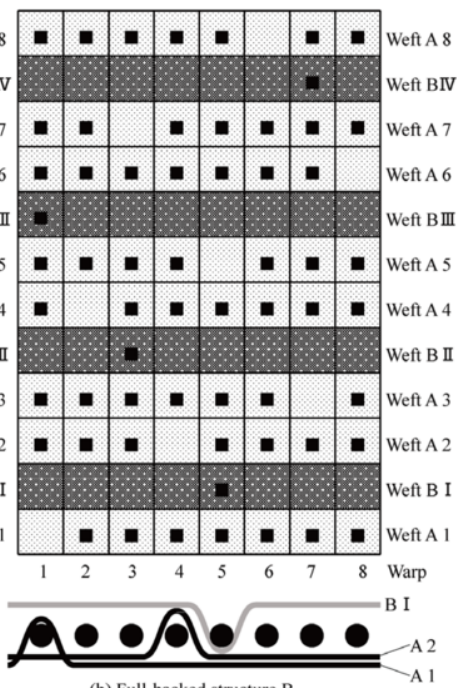

(b) Full-backed structure B

Figure 2. Design principle for 2:1 weft-backed structure.

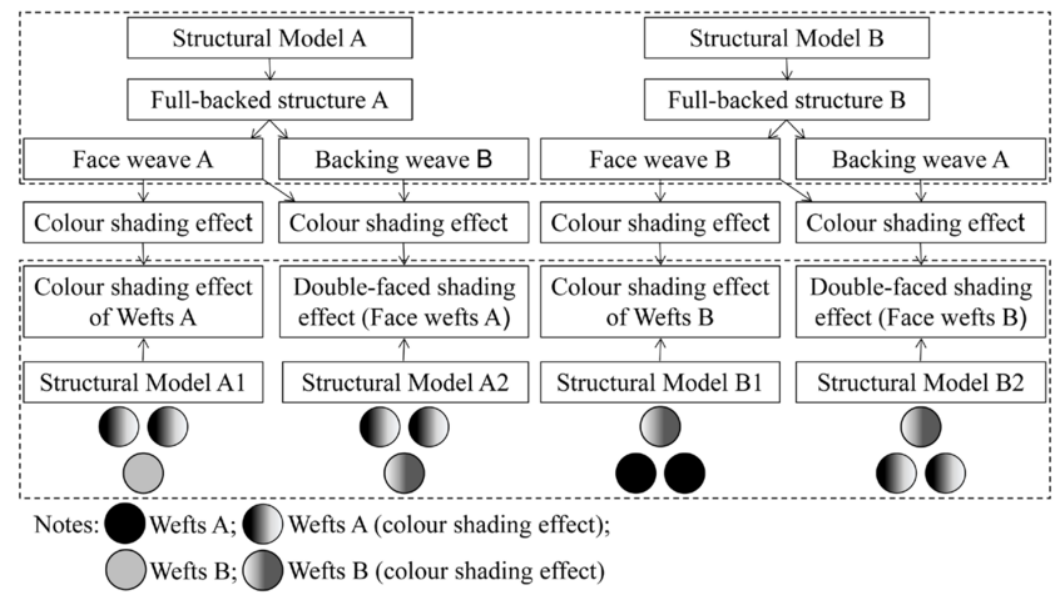

Figure 3. Design process of four digital structural models.

\section{Digital design method for jacquard fabric with 2:1 weft-backed structure}

Based on the design principle of four digital structural models proposed above, four databases of compound structure can be designed to achieve four kinds of colour shading effects. In practice, four databases of compound structure can be used individually or in combination to create a variety of digital jacquard fabric products with different colour shading effects. The combined application of models A1 and B1 is taken as an example to illustrate the design approach as follows. 


\subsection{Structural design}

The design process for model A1 is shown in Figure 4. Firstly, face weave A and backing weave $\mathrm{B}$ were designed according to the covering principle [7], as shown in Figure 5 (a) and (b) respectively. The design of the full-backed points $\mathrm{A}$ is the key to ensuring that Wefts A can cover Wefts B when the colour shading effect of Wefts A is shown on the face of the fabric. The full-backed points A (Figure 5(c)) was designed by first extending each weft of the backing weave B upwards and then replicating it along the warp and weft directions until its weave repeat was equal to that of the face weave A.

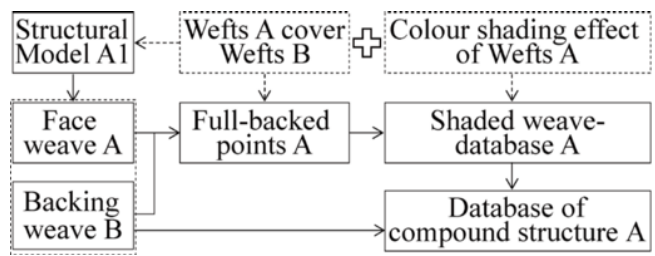

Figure 4. Digital design process for model A1.

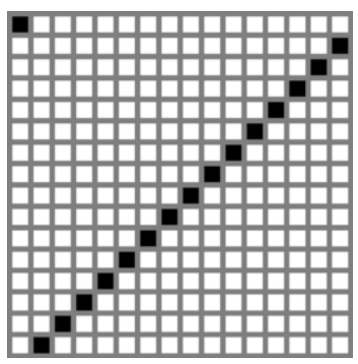

(a) Face weave A

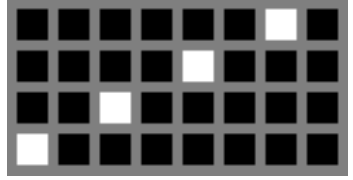

(b) Backing weave B

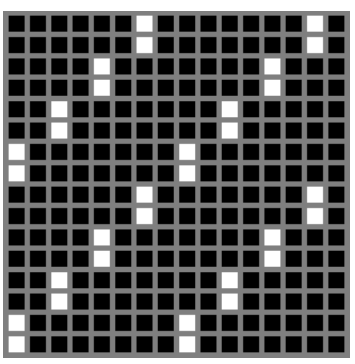

(c) Full-backed points A

Figure 5. Face weave A, backing weave B and full-backed points A.

To achieve the colour shading effect of Wefts A, face weave A was added $M$ weave points once along the weft direction. During the enhancement, it skipped when the weft points in the full-backed points A were encountered. $M$ represents the added value of weave points. In doing so, shaded weave-database A with 13 shaded effects was formed (Figure 6). The database of compound structure A was established by combining each weave in the shaded weave-database A with the backing weave B in a 2 to 1 ratio along the warp direction. The total number of weaves in the databases of compound structure A determines the maximum number of colours that can be shown by Wefts B. The maximum and minimum number of weaves in the databases of compound structure A were calculated by equations (1) and (2) respectively.

$$
\begin{aligned}
& N_{\text {max }}=R\left(R-J_{A}-1\right) \\
& N_{\text {min }}=R-J_{A}-1
\end{aligned}
$$

Where $N_{\max }$ and $N_{\min }$ represent the maximum and minimum number of weaves 
respectively; $R$ represents the number of warp weave repeat in face weave; $J_{\mathrm{A}}$ represents the number of weft points per weft in the full-backed points A.
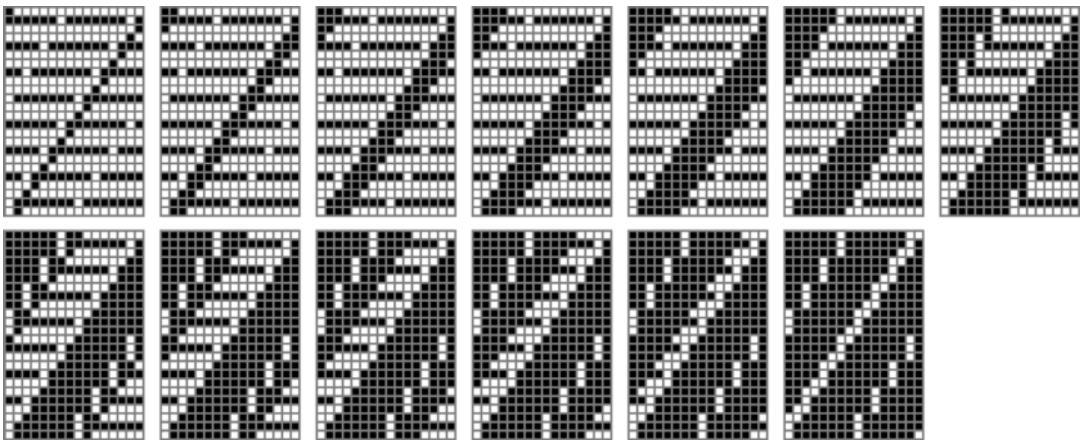

Figure 6. Minimum database of compound structure A.

Similarly, the digital design process for model B1 is shown in Figure 7. It contains the design of face weave $\mathrm{B}$, backing weave $\mathrm{A}$, full-backed points $\mathrm{B}$, shaded weavedatabase $\mathrm{B}$ and databases of compound structure $\mathrm{B}$. The design of the full-backed points B is the key to ensuring that Wefts B can cover Wefts A when the colour shading effect of Wefts B is shown on the face of the fabric. The full-backed points B (Figure 8(c)) was designed by first decomposing the backing weave A into 2 weaves according to odd and even wefts, then overlaying the weft points of these 2 weaves and finally copying it along the warp and weft directions until its weave repeat is equal to that of the face weave B. The shaded weave-database B and the databases of compound structure B (Figure 9) were created by a design approach similar to that of model A1. The maximum and minimum number of weaves in the databases of compound structure B were calculated by equations (3) and (4) respectively.

$$
\begin{aligned}
& N_{\text {max }}=\frac{R}{2}\left(R-J_{B}-1\right)+1 \\
& N_{\text {min }}=R-J_{B}
\end{aligned}
$$

Where $J_{\mathrm{B}}$ is the number of weft points per weft in the full-backed points B.

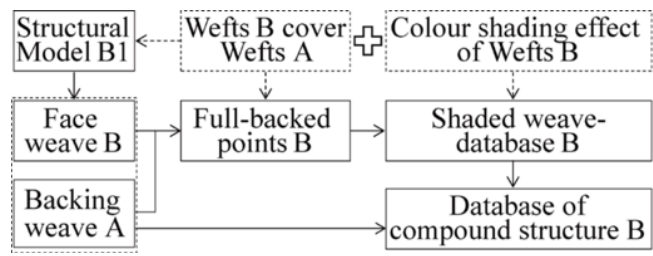

Figure 7. Digital design process for model B1. 


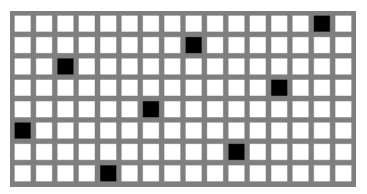

(a) Face weave B

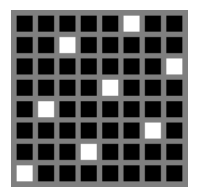

(b) Backing weave A

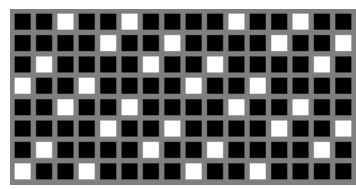

(c) Full-backed points B

Figure 8. Face weave B, backing weave A and full-backed points B.
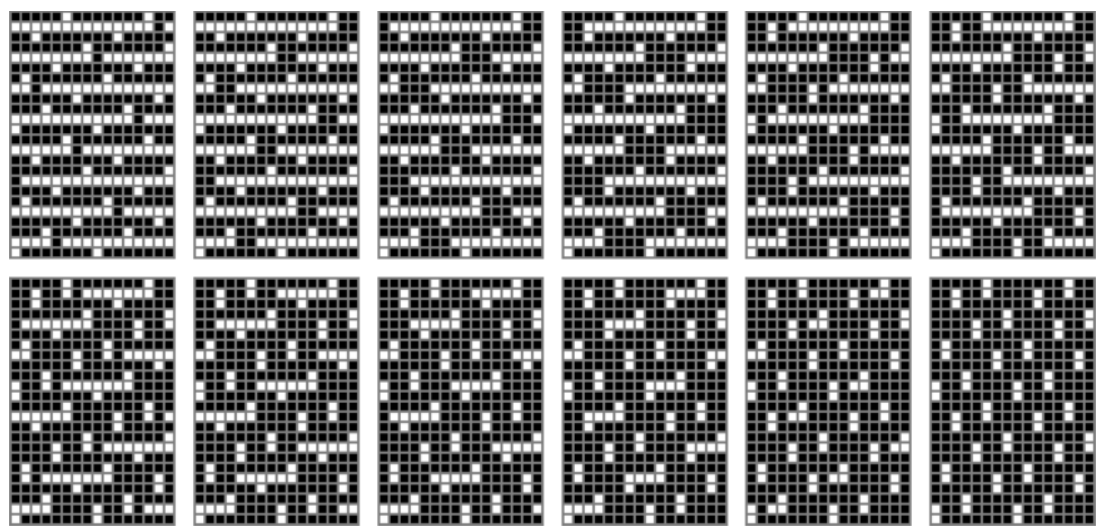

Figure 9. Minimum database of compound structure B.

\subsection{Design Practice}

Digital jacquard fabric with the colour shading effects of Wefts A and Wefts B can be created by the 2 databases of compound structure mentioned above. The digital pattern was made up of 2 shaded colours with a grey level less than or equal to 13 and 12 respectively. The resulting point paper (Figure 10) was a combination of white weft points and black warp points, which correspond to 0 and 1 in binary, respectively. After that, a colourful fabric pattern was created by weaving.

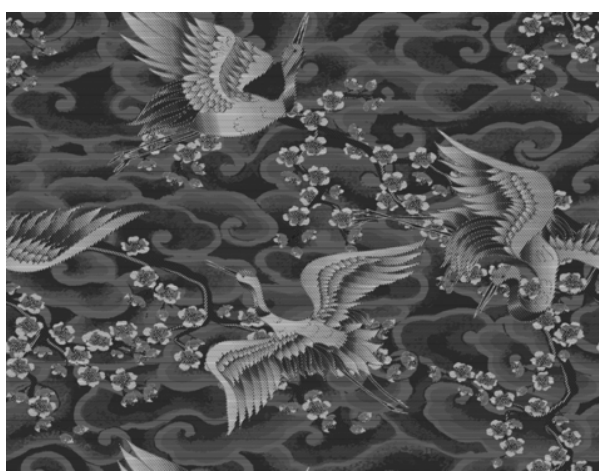

(a) Overall view of point paper

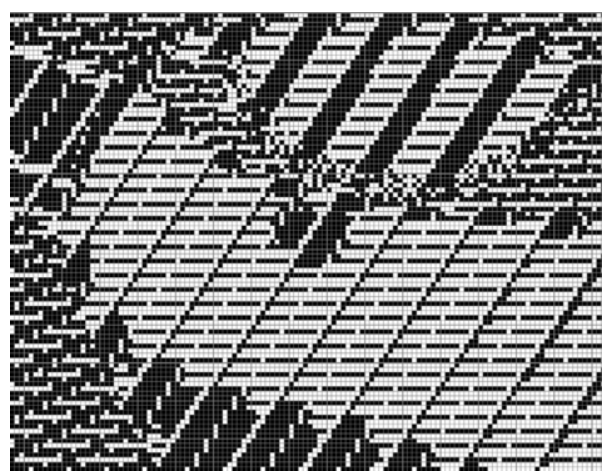

(b) Details of point paper

Figure 10. Point paper and its details. 
The face effect and the colour shading effect of the fabric are shown in Figure 11. 2 colour shading effects from white to black and from red to black were achieved on the face of the fabric. The shaded colour from white to black was suitable for expressing the main motifs. In this part, 2 sets of white wefts on the face covered 1 sets of red wefts on the reverse, which not only improved the fullness and delicacy of the shaded colour on the face, but also saved the usage of Wefts B on the reverse. The shaded colour from red to black was suitable for the expression of minor motifs. The maximum number of fabric colours was calculated by equation (5).

$$
N_{\max }=(1)+(3)=\frac{R}{2}\left(3 R-2 J_{A}-J_{B}-3\right)+1
$$

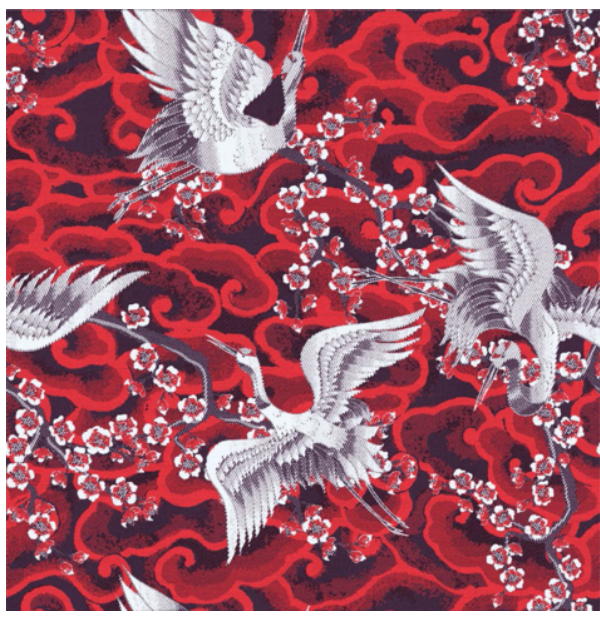

(a) Face effect of fabric

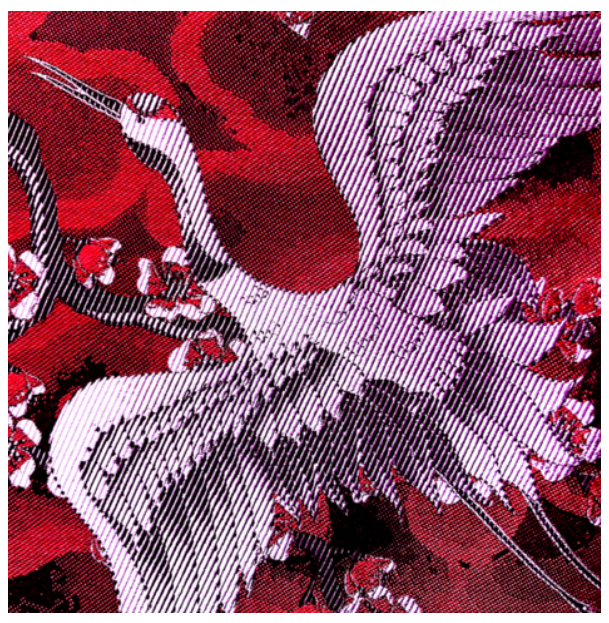

(b) Colour shading effect of fabric

Figure 11. Face effect and colour shading effect of digital jacquard fabric based on model A1 and model B1.

\section{Conclusion}

Based on digital jacquard technology, the design principle and method of digital jacquard with 2:1 weft-backed structure was proposed. Four digital structure models proposed in this paper can be used either individually or in combination in practice. It lays a theoretical foundation for the creation of digital jacquard fabrics with colour shading effects. The design practice showed that the combined applications of model A1 and model B1 effectively improved the colour expression and yarn utilisation. The resulting digital jacquard fabric had 2 colour shading effects, i.e., the colour shading effects of Wefts A and Wefts B. The maximum number of fabric colors was $\left(\frac{R}{2}\left(3 R-2 J_{A}-J_{B}-3\right)+1\right)$. The results of this paper expand the innovative design ideas for digital jacquard fabrics and provide a reference for the structural innovation and variety creation of traditional jacquard fabrics. Further research on the digital 
design method of models A2 and B2 will be needed to complete the design research framework for digital jacquard fabrics with 2:1 weft-backed structure.

\section{Acknowledgements}

Authors are thankful for providing the funds by the 2018 "the Light of Textile" Foundational Applied Research Project [grant No. J201802].

\section{References}

[1] Xiaoping QIAN. A complete collection of Chinese silk: Three Famous Brocades. 2014: Beijing: China Textile Publishing House; p. 203-213.

[2] Jiu ZHOU. Analysis on variety and aesthetic characteristics of three famous brocades in ancient China. Journal of Silk. 2018 Apr; 55(4): 93-105.

[3] Jiu ZHOU, Linlin BAI. Design research and practice on gradient weft-full-backed structure. Journal of Textile Research. 2018 Jan; 39(1): 32-38.

[4] Aidan ZHANG, Jiu ZHOU. Design principle of double face jacquard fabric with pattern effect based on double weft. Journal of Textile Research. 2011 Dec; 32(12): 38-41.

[5] Ping GU. Fabric weaves and structures. 2010: Shanghai: Donghua University Press; p. 83-84.

[6] Jiu ZHOU, Lina DUAN, Yongjian TU. Design principle and method of color gradient expression on double-warp double-weft jacquard fabric. Journal of Textile Research. 2016 Jun; 37(6): 36-41.

[7] Xiaoping QIAN. Discuss backed weaves' appearance of grounding. Journal of silk. 1980 Feb; 28(2): 59-62. 